\title{
Chronic pain and pain relief by extracorporeal shock wave therapy
}

\author{
Othmar J. Wess
}

Received: 28 December 2010/Accepted: 28 March 2011/Published online: 12 April 2011

(c) Springer-Verlag 2011

To the Editor:

My paper: A neural model for chronic pain and pain relief by extracorporeal shock wave treatment (Urol Res (2008) 36:327-334) [1] provoked a vigorous offence by $\mathrm{Ch}$. Schmitz and DePace (Letter to the Editor: Pain relief by extracorporeal shockwave therapy: an update on current understanding, Urol Res (2009) 37:231-234) [2] which requires a clarifying reply.

\section{What is the concept and content of the neural model presented?}

The original paper outlines a new concept of chronic pain on the basis of neural pain memory. The basic idea is the establishment of "pathologic" reflex patterns linking sensory input with motor output nerve signals during chronification of pain sensation. The neurological mechanism behind is hypothesized to be based on the plasticity of synaptic junctions. The working mechanism of shock wave therapy is hypothesized to erase the "pathologic" links between sensory input and motor output by hyper stimulation through shock wave stimuli (for details see original paper).

Presenting a hypothesis as published enables the scientific community to test the hypothesis against real biological findings. Scientific hypotheses in general need to be detailed and substantiated to enable basic tests for possible falsification, whereas a general verification is impossible, see e.g., Popper [3]. Nevertheless, a detailed and substantiated hypothesis/theory is of high value providing a basis

O. J. Wess $(\bowtie)$

Storz Medical AG, Tägerwilen, Switzerland

e-mail: wess.othmar@storzmedical.com for realistic predictions which, according to experience, come true as long as attempts for falsification are not successful (Popper, ibid.). The purpose of my paper is to present a new model of chronic pain in general and of chronic pelvic pain (CPP), in particular, as well as developing a possible neural working mechanism of shock wave therapy. The hypothesis is based on the neural functions of the brain and is open for critical review and substantiated attempts of falsification. This is a widely accepted scientific method to achieve objective knowledge of empirical sciences (see Popper, ibid.) at least the method I prefer.

\section{What is the concept and content of the Letter of Schmitz and DePace?}

First of all I wonder why the Letter to the Editor was published since it does not focus on the matter of my hypothesis at all but presents offending speculations of questioning my competence and earnestness to publish a scientific hypothesis. Instead, Schmitz and DePace take the opportunity to elaborate on a separate subject of molecular mechanisms of ESWT, a field, one of the authors, Schmitz, has worked upon. Molecular mechanisms are the physiological substructure of biological functions including neural activities but are not the subject of the original paper. Neither the presented claims, papers and personal speculations are focused on substantial details of my hypothesis nor could I come to the realization that any attempt has been made to falsify my hypothesis, in order to be a decent scientific approach.

The authors, however, exhibit personal critics on my paper mainly in four different aspects (see below) in an apparent expert manner including imputations with respect to an unfair and hidden motivation due to my affiliation 
with Storz Medical AG without any objective substance. Since it had been published, I will briefly comment on this:

1. A warning to apply shock wave therapy to indications such as chronic pelvic pain and angina pectoris "We believe, a clear warning should be expressed to your readership (...) p. 231, 1st column, lines 9-10 (...) chronic pelvic pain syndrome or angina pectoris can be regarded only experimentally to date (...) or for that matter, anecdotally. The casual use of ESWT in the treatment of angina pectoris could result in unwanted side effects such as embolism or even severe damage of lung." p. 231, 1st column, lines $22 \mathrm{ff}$.

2. Refusal of the involvement of higher levels of the central (CNS) and peripheral nervous system (PNS) "ESWT is not used in the international peer-reviewed literature to treat pain without underlying anatomical disorders (original paper), and ESWT cannot be regarded no more focused on specific organs under pain but on pain memory (original paper)".

3. Neglect of relevant literature "Our major concern, however, is linked to Section Associative memory model for establishing reflex functions (original paper) of Dr. Wess' article. This section outlines in one and half pages, a hypothesis of so-called associative pain memory, without so much as a reference to the literature" p. 231, 2nd column, lines 2-7.

4. Imputation of hidden motivation "One can only speculate as to Dr. Wess' motivation to publish his hypothesis (...)." "An interesting indication in this regard might emerge from the fact that Dr. Wess is affiliated with Storz Medical AG (Tägerwilen, Switzerland), the manufacturer of several shock wave systems (among them the D-ACTOR 200 marketed in the USA (...). The Food and Drug Administration (FDA) classifies extracorporeal shock wave devices as Class III, higher risk devices", p. 232. 2nd column, lines 37-48. "In contrast, the extracorporeal shock wave system D-ACTOR 200 has been presented to FDA as Class I medical device”, p. 233, 1st column, lines 32-34.

Response to 1 The medical indications chronic pelvic pain syndrome (CPPS) or angina pectoris (as well as others) are, in fact, clinically used and taken as an example for the surprisingly wide variety of successful application of extracorporeal shock wave therapy (ESWT). They are used as introduction into the subject of my paper for clarification of the wide impact of ESWT, not limited to a single specific disease. The purpose of the paper is not developing "guidelines for the application of ESWT" but to refer to medical facts. Meanwhile, there are several prospective, randomized, double-blinded controlled studies supporting safety and efficacy of the mentioned indications [4, 5].
Both, CPPS and Angina pectoris (as well as others), are cleared by European Authorities (CE-Mark) for specific shock wave devices such as the DUOLITH SD1 and the MODULITH SLC from Storz Medical AG. (Both devices are considered "shock wave devices" in a physical sense of the term "shock waves" whereas this does not apply to the D-ACTOR 200.) Since any therapeutic procedure needs to be performed by medical personnel in a professional manner, a specific warning imputes an unjustifiable and careless behavior of the author of the original paper which is inappropriate.

Response to $2 \mathrm{Schmitz}$ and DePace oppose against the term: pain without underlying anatomical disorders (original paper) and replace it by "anatomically defined" diseases (p. 232 left column, lines 3 and 30). If we consider chronic pain without underlying anatomical disorders, we will exclude pain diseases due to acute injuries or other persistent local reasons such as tumors or acute external stimuli such as excessive heat, cold, electrical, mechanical or chemical and other pain generating stimuli. Chronic pain is understood as pain no longer acutely caused by one of the mentioned reasons. There are several chronic pain diseases no longer maintained by acute injuries or external pain generating stimuli (which often have been the original cause or trigger of pain during the acute phase), but which persist for a long period of time after the initial injury or the stimulus has been dissolved.

The European Association of Urology (EAU) defines chronic pain (in relation, e.g., to CPPS) as follows [6]:

Acute or chronic pain Pain may also be described as either acute or chronic pain:

- Acute pathological pain has an acute onset and is shortlived, usually less than 1 week or so, and is associated with tissue trauma, e.g., following surgery. Transient acute pain may also be caused by acute nerve injury, e.g., local injury to the ulnar nerve from hitting the elbow. Although the mechanisms of acute and chronic pain may overlap, the mechanisms of acute pain resolve quickly in contrast to chronic pain.

- Chronic (also known as persistent) pain occurs for at least 3 months. However, the mechanisms involved are more important than the duration of the pain. Chronic pain is associated with changes in the central nervous system (CNS), which may maintain the perception of pain in the absence of acute injury. These changes may also magnify perception so that non-painful stimuli are perceived as painful (allodynia), while painful stimuli are perceived as more painful than expected (hyperalgesia). The bladder provides a good example of how changes in the CNS affect sensory perception. An acute pain insult to the bladder can produce functional changes within the CNS, so that pain persists even 
after removal of the stimulus. These central functional changes may also be associated with a dysaesthetic (unpleasant sensation) response; for instance, mild distension or stimulation of the bladder by urine not normally perceived may produce the urge to urinate. Furthermore, core muscles, including pelvic muscles, may become hyperalgesic with multiple trigger points, while other organs may also become sensitive, e.g., the uterus with dyspareunia and dysmenorrhea, the bowel with irritable bowel symptoms. The spread of abnormal sensory responses between the organs and musculoskeletal system is a well-described consequence of the CNS changes and a crucial cause of complex chronic pelvic pains. Functional abnormalities such as urinary retention may also occur. Chronic pain is associated with various psychological responses, partly due to the long duration of the pain and partly due to neuroplasticity of the CNS. Chronic pain inhibits feelings, emotions, thinking and reactions, while reduced mobility and inhibited physiological functions restrict social interactions and work. Although there are established management strategies, pain is often undertreated because many clinicians have a poor understanding of the principles of pain therapy. Efforts are needed to improve this situation. When appropriate, management should be both holistic and multidisciplinary.

Often muscle and vessel spasms are associated with chronic pain resulting in reduced circulation and malnutrition of the affected organ as outlined. In consequence to persistent malnutrition and reduced circulation, however, impairment of organ functions may occur later.

The involvement of the central nervous system in the development of chronic pain is recognized by several authors [7-9] and can be considered state of the art. The presented hypothesis of associative pain memory is based on these general ideas. It does not only focus on local biochemical effects but, complementary, on higher levels of the peripheral and central nervous system. The innovative part of the hypothesis is the proposed working mechanism of chronification as outlined on the basis of a holographic brain model in some detail which, in turn, offers a new hypothesis for the working mechanism of ESWT for pain relief. This is the purpose of the original paper as outlined. To avoid further misinterpretations, I would like to remind the reader of the original paper: Shock waves are applied extracorporeally, by coupling the therapy head of the device to the painful area [original paper, p. 328, 1st Column, lines 4-6)]. The term no more focused on specific organs under pain but on pain memory (original paper) does not stand for a direct exposure of shock waves to the brain but to the end organ itself, activating the specific (organ related) sensory and motor nervous pathways to and from the brain to the organ under treatment. Insofar, the therapeutic approach, according to the pain memory concept, is no more focused on specific organs under pain but on pain memory (original paper).

Finally, I do not deny molecular mechanisms at the end organ triggered by ESWT (mechano-transduction). But that is not the issue of my paper. On the contrary, the painful shock wave impact results in generation of strong neural action potentials which are traveling from the impact area to higher levels of the CNS. This type of mechano-sensory transduction is considered to be a prerequisite for ESWT affecting pain memory. This, in turn, could be an explanation for the reduced effect of ESWT when applied under local anesthesia, since strong nerve signals are blocked and cannot reach higher levels of the CNS.

As outlined above, the unquestioned involvement of molecular mechanisms does not exclude memory mechanisms of the CNS and they are not sufficient enough to understand chronic pain and pain relief by ESWT. Therefore, restriction to molecular mechanisms only, cannot be seen as an update on current understanding but rather a step backwards.

Response to 3 Memorizing, the subject of the original paper outlines "A neural model for chronic pain and pain relief by extracorporeal shock wave treatment". Accordingly, references $12-25$ (see original paper) relating to the most relevant publications with respect to the outlined neural model are cited.

Schmitz and DePace, however, criticise for not quoting their own and other papers regarding to local mechanisms of liberation of biochemical drugs and their influence on the healing process initiated by ESWT. The list of literature presented in the Letter by Schmitz and DePace is neither neglected nor considered to be irrelevant. Citations 1-11 of the original paper related to the local (biochemical) effects published by several authors in an exemplary manner, without focussing explicitly on the papers listing Schmitz as co-author.

I am well aware of shock wave effects at the end organ such as liberation of nitric oxides (NO), vascular endothelial growth factors (VEGF) [10], substance P [11] and others. Temporary increase in circulation and metabolism is recognized. Relief of chronic pain, however, cannot be solely restricted to biochemistry mechanisms at the end organ by dogmatic insistence on molecular mechanisms as published by Schmitz et al. The reported liberation of substance P, e.g., does not falsify my hypothesis of shock wave interaction with pain memory, however, supports the idea. In my opinion, permanent healing of chronic pain diseases is better understood by taking additional (complementary) mechanisms into account, affecting chronic pain memory as, e.g., elaborated and published in the original paper. 
Although Schmitz and DePace seem to believe in a molecular model of pain relief, being exclusively the one and only, there is no scientific reason to disclaim alternative concepts as long as they could not falsify them.

Response to $4 \mathrm{It}$ appears to be the main issue for Schmitz and DePace to exclude a competitive product (D-ACTOR 200, Storz Medical) from the American market by claiming the D-ACTOR 200 being incorrectly presented to the FDA. "Dr. Wess' hypothesis (...) should be evaluated cautiously against the fact that the extracorporeal shock wave system D-ACTOR 200 manufactured by Storz Medical AG (Dr. Wess' affiliation) has not been presented to FDA as a Class III extracorporeal shock wave device (...)". In other words, The D-ACTOR 200 is claimed to be an unproven extracorporeal shock wave device, which would require an IDE study according to a correct classification (Class III) same as the competing DolorClast by EMS.

With these statements, Schmitz and DePace introduce marketing aspects which have nothing to do with my hypothesis.

It does not become apparent to me why Schmitz and DePace link my hypothesis to the product classification according to the American Food and Drug Administration (FDA). FDA's criteria for market clearance of medical products in the US are "safety and efficacy". Up to my knowledge, there is no reason why my hypothesis has any impact on the FDA's judgment regarding either one of the two criteria or why it should have any impact on the market clearance of the D-ACTOR 200 in the US. Moreover, the hypothesis was orally presented at several congresses as early as 2001 (Mainz) [12], 2004 (Kaoshiung) [13]), 2005 (Vancouver) [14], years before the D-ACTOR 200, was introduced in the market in 2008. Schmitz and DePace impute the classification issue being the hidden motivation of my hypothesis. I forcefully reject this unfounded speculation.

Schmitz and DePace complain about the D-ACTOR 200 not being classified as (orthopedic) "extracorporeal shock wave device" (Class III), whereas the Swiss DolorClast (EMS Electro Medical Systems, Nyon Switzerland) the company, Schmitz and DePace have been affiliated with, was classified Class III (requiring an extensive and costly IDE study). There is a simple reason: the D-ACTOR 200 is not a shock wave device in the physical sense. This holds also true for the DolorClast of EMS [15] which is substantially equivalent to the D-Actor 200 even if it had been presented to FDA as Class III device. According to the clearance issues, it makes a crucial difference with respect to market access, notably in the US.

One should keep in mind that the D-ACTOR 200 was not mentioned in my original paper at all and was no subject thereof. If at all, specific shock wave devices in a physical sense such as the DUOLITH SD1 and the MODULITH SLC of Storz Medical AG may be mentioned in this regard.

The background of my hypothesis roots back to the late 1990s (first presented in 2001) and is based on the concept of a holographic brain model published in the 1970s by myself and other authors (see references $1-12$ of the original paper).

Finally, Schmitz and DePace express a warning against my hypothesis without any falsification thereof and stoke fears regarding the safety of the D-Actor device:

“Dr. Wess' hypothesis (...) should be evaluated cautiously against the fact that the extracorporeal shock wave system D-ACTOR 200 manufactured by Storz Medical AG (Dr. Wess' affiliation) has not been presented to FDA as a Class III extracorporeal shock wave device but rather as Class I device, being similar in function and purpose to Therapeutic Massagers."

Schmitz and DePace do not only ignore the fact that the D-Actor 200 is not a shock wave device (as well as the EMS Dolorclast, see above), but also impute a possible risk of the device without providing any reason whatsoever. FDA's classification, however, cannot be taken for proof: since firstly, the D-ACTOR is not a shock wave device as mentioned and secondly, not every shock wave device is classified Class III by the FDA. Shock wave lithotripsy devices cleared for use in urology (kidneys stone lithotripter) are classified Class II although exposing well-perfused kidney tissue with approximately ten times higher shock wave energy as specific "orthopedic shock wave lithotripters" which are classified Class III. The inconsistent use of the term "shock waved device" by FDA does not qualify a "none shock wave device" as a real "shockwave device". FDA, although being an US-governmental institution, is not to be acknowledged as the authority for standardization such as the National Institute of Standards and Technology (NIST) and cannot define ordinary pressure waves (as used by the EMS Dolorclast and the Storz Medical D-Actor) as "shock waves" in a physical sense. The Urological Research Journal might not the right place to discuss these market clearance issues. However, they were brought up by the Letter of Schmitz and DePace and requested a response.

\section{Conclusion}

The Letter to the Editor of Schmitz and DePace did not do justice to the issue of my original paper.

It is to be hoped that my hypothesis gets a fair chance to be critically reviewed by the scientific community. The hypothesis does not deserve a disparaging attack based on the competition and marketing issues. Critical and detailed 
evaluation of the hypothesis presented may not only guide the way for a better understanding of chronic pain phenomena but may also develop a basis for better treatment options of CPPS and other chronic pain diseases.

\section{References}

1. Wess OJ (2008) A neural model for chronic pain and pain relief by extracorporeal shock wave treatment. Urol Res 36:327-334. doi:10.1007/s00240-008-0156-2

2. Schmitz Ch, DePace R (2009) Pain relief by extracorporeal shockwave therapy: an update on current understanding. Urol Res 37:231-234. doi:10.1007/s00240-009-0190-8

3. Popper K (1994) Logik der Forschung: Zehnte, verbesserte und vermehrte Auflage, J.C.B. Mohr (Paul Siebeck) Tübingen. Original: Logik der Forschung, Julius Springer Verlag. Wien (1934). English: The Logic of Scientific Discovery, Hutchinson, London 1959

4. Zimmermann R et al (2009) Extracorporeal shock wave therapy for the treatment of chronic pelvic pain syndrome in males: a randomised, double-blind, placebo-controlled study. EURURO 56:418-424

5. Kikuchi Y et al (2010) Double-blind and placebo-controlled study of the effectiveness and safety of extracorporeal cardiac shock wave therapy for severe angina pectoris. Circ J 74:589-591

6. Fall M et al (2010) EAU guidelines on chronic pelvic pain. Eur Urol 57:35-48
7. Melzack R (2001) Pain and the neuromatrix in the brain. J Dent Educ 65(12):1378-1382

8. Melzack R (1999) From the gate to the neuromatrix. Pain (Suppl 6):121-126

9. Baranowski A (2009) Chronic pelvic pain. Best Pract Res Clin Gastroenterol 23:61-593

10. Wang CJ, Wang FS, Yang KD, Weng LH, Hsu CC, Huang CS, Yang LC (2003) Shock wave therapy induces neovascularization at the tendon-bone junction. A study in rabbits. J Orthop Res 21:984-989

11. Hausdorf J, Lemmens M, Kaplan S, Marangoz C, Milz S, Odaci E, Korr H, Schmitz C, Maier M (2008) Extracorporeal shockwave application to the distal femur of rabbits diminishes the number of neurons immunoreactive for substance $\mathrm{P}$ in dorsal root ganglia L5. Brain Res 1207:96

12. WessO (2001) Einfluss der ESWT auf das assoziative Schmerzgedächtnis, Muskuloskeletale Stosswellentherapie, DreiLänder-Treffen der österreichischen, schweizer und deutschen Fachgesellschaften 30. und 31. März 2001 Klinikum der Johannes Gutenberg Universität Mainz

13. Wess O (2004) Hypothesis towards associative pain memory and pain management by shock wave therapy, in Abstracts 7 th ISMST Congress, Kaohsiung, Taiwan. http://www.ismst.com

14. Wess O (2005) Reorganization of pathological control functions of memory-a neural model for tissue healing by shock waves (A). J Acoust Soc Am 117(4):2383

15. Cleveland RO et al (2007) Acoustic field of a ballistic shock wave therapy device. Ultrasound Med Biol 33(8):1327-1335 